\title{
Temperature-dependent structural behaviour of samarium cobalt oxide
}

Jiang $^{1}$

Matthew R. Rowles ${ }^{\mathrm{a}), 1}$, Cheng Wang ${ }^{1}$, Kongfa Chen ${ }^{1}, \mathrm{Na} \mathrm{Li}^{1,2}$, Shuai $\mathrm{He}^{1}$ and San-Ping

${ }^{1}$ Fuels and Energy Technology Institute, Curtin University, GPO Box U1987, Perth WA, Australia, 6185

${ }^{2}$ College of Science, Heilongjiang University of Science and Technology, Harbin 150022, China

The crystal structure and thermal expansion of the perovskite samarium cobalt oxide $\left(\mathrm{SmCoO}_{3}\right)$ has been determined over the temperature range 295 to $1245 \mathrm{~K}$ by Rietveld analysis of X-ray powder diffraction data. Polycrystalline samples were prepared by a sol-gel synthesis route followed by high-temperature calcination in air. $\mathrm{SmCoO}_{3}$ is orthorhombic (Pnma) at all temperatures and is isostructural with $\mathrm{GdFeO}_{3}$. The structure was refined as a distortion mode of a parent $P m-3 m$ structure. The thermal expansion was found to be non-linear and anisotropic, with maximum average linear thermal expansion coefficients of 34.0(3) $\times 10^{-6} \mathrm{~K}^{-1}, 24.05(17) \times 10^{-6} \mathrm{~K}^{-}$ ${ }^{1}$, and $24.10(18) \times 10^{-6} \mathrm{~K}^{-1}$ along the $a, b$, and $c$ axes, respectively, at between 814 and $875 \mathrm{~K}$.

Key words: structure determination; thermal expansion; solid oxide fuel cell

\section{INTRODUCTION}

Cobaltite perovskite oxides such as ( $\mathrm{La}, \mathrm{Sr}) \mathrm{CoO}_{3-\delta}$ (LSC), ( $\left.\mathrm{Sm}, \mathrm{Sr}\right) \mathrm{CoO}_{3-\delta}$ (SSC) and (Ba,Sr) $(\mathrm{Co}, \mathrm{Fe}) \mathrm{O}_{3-\delta}(\mathrm{BSCF})$ are mixed ionic/electronic conductors with high electrocatalytic activity to promote oxygen reduction required for their use as cathodes in solid-oxide fuel cells (Sun et al. 2009). Compared to LSC, SSC, with a smaller cation in the A site (Sm vs La), has been demonstrated to show much better oxygen surface exchange kinetics (Fukunaga et al. 2000). Therefore, SSC has been extensively investigated as a promising cathode material for intermediate-temperature solid oxide fuel cells (Dong et al. 2012; Ishihara et al. 1998; Tu et al. 1997; Xia et al. 2002; Yang et al. 2008).

The room-temperature structure of the SSC end-member, $\mathrm{SmCoO}_{3}$, has been studied previously in the context of solid-solution series with Ni (Pérez-Cacho et al. 2000), Fe (Kharko et al. 2014), and Sr (Tu et al. 1997), which, in all cases, was reported as the orthorhombic Pbnm space-group (standard setting Pnma, no. 62 (Hahn 1995)). Tu et al. (1997) does report the rate of $\mathrm{SmCoO}_{3}$ thermal expansion up to $1000^{\circ} \mathrm{C}$, however, this was measured on bulk, polycrystalline specimens, and reveals no information about any thermal expansion dependence on crystallographic parameters.

The aim of our research program is to investigate the atomic structure, thermal expansion behaviour, and electrochemical properties of various perovskites in order to correlate their structural parameters and behaviour as a solid-oxide fuel cell cathode. This work reports the temperature-dependent structure of $\mathrm{SmCoO}_{3}$ from room temperature to $1245 \mathrm{~K}$.

a) To whom correspondence should be addressed. Electronic mail: matthew.rowles@curtin.edu.au 


\section{EXPERIMENTAL}

\section{A. Sample Preparation}

$\mathrm{SmCoO}_{3-8}$ powder was synthesised by the combined citrate and ethylenediaminetetraacetic acid (EDTA) complexing method (Chen et al. 2016; Wang et al. 2009; Shao et al. 2012). The raw chemicals were $\mathrm{Sm}\left(\mathrm{NO}_{3}\right)_{3} \cdot 6 \mathrm{H}_{2} \mathrm{O}$ (99\%, Sigma-Aldrich), $\mathrm{Co}\left(\mathrm{NO}_{3}\right)_{2} \cdot 6 \mathrm{H}_{2} \mathrm{O}(98.0 \%$ to $102.0 \%$, AlfaAesar), citric acid (99.5\%, Chem Supply), EDTA (99\%, Acros Organics) and ammonia (28\%, Ajax Finechem). The molar ratio of metal ions/citric acid/EDTA was 1:1.5:1. The resultant powder was calcined at $900{ }^{\circ} \mathrm{C}$ in air for $2 \mathrm{~h}$.

\section{B. In situ synchrotron $\mathrm{X}$-ray powder diffraction}

High-resolution X-ray powder diffraction patterns were collected using the Powder Diffraction beamline (Wallwork et al. 2007) at the Australian Synchrotron. A monochromatic X-ray beam with an energy of $15.5108 \mathrm{keV}$ (determined from $\mathrm{LaB}_{6}$ (NIST SRM 660b)) was used in order to keep away from the Sm L absorption edge to avoid spurious detector effects arising from specimen fluorescence. Data were collected over the angular range $3^{\circ}$ to $83^{\circ} 2 \theta$ using an array of Mythen position sensitive detector modules (Schmitt et al. 2003). To eliminate the gaps between the individual Mythen modules, the diffraction patterns were acquired in pairs with a goniometer rotation of $0.5^{\circ} 2 \theta$ between each pattern, and merged using the program CONVAS2 (Rowles 2012). The $\mathrm{SmCoO}_{3}$ powder was loaded in a $0.5 \mathrm{~mm}$ quartz capillary and mounted in DebyeScherrer geometry. The capillary was continuously rotated about its long axis at $\sim 1 \mathrm{~Hz}$ in order to provide even heating, improve particle statistics, and ensure accurate relative peak intensities.

The specimen was heated using a hot-air blower at a constant rate of $8 \mathrm{Kmin}^{-1}$ from room temperature up to $1245 \mathrm{~K}$ before cooling as rapidly as possible. The temperature was controlled by a thermocouple placed between the nozzle of the hot-air blower and the capillary. The specimen temperature was calibrated (Stinton and Evans 2007) using the known thermal expansion coefficients of corundum (Taylor 1984) applied to NIST SRM 676a. In situ diffraction patterns were collected for $45 \mathrm{~s}$ in each goniometer position, with approximately $13 \mathrm{~s}$ between each diffraction pattern for goniometer movement, for the duration of the experiment.

\section{RESULTS AND DISCUSSION}

Select diffraction patterns over the entire temperature range were indexed by the SVDIndex algorithm (Coelho 2003) in TOPAS (Bruker AXS 2014) and were all found to be consistent with the orthorhombic space-group Pnma, as has been found in previous room-temperature studies (Kharko et al. 2014; Pérez-Cacho et al. 2000; Tu et al. 1997). There were several unidentified minor impurity peaks present, but they were able to be discounted, as their thermal expansion behaviour was markedly different to that of the main perovskite phase.

All diffraction data were analysed by the Rietveld method (Rietveld 1969) as implemented in TOPAS. Refined parameters included background coefficients, specimen displacement (Scarlett et al. 2011), lattice parameters, profile coefficients, atomic position coordinates, and isotropic atomic displacement parameters. The zero offset was constrained to be the same over all patterns. The effect of specimen absorption and capillary geometry on peak intensities and profiles was modelled using a capillary aberration function based on the work of Sabine et al. (1998). The temperature calibration data were parametrically refined (Stinton and Evans 2007) to give the actual specimen temperature, which was then used unchanged. The isotropic displacement

a) To whom correspondence should be addressed. Electronic mail: matthew.rowles@curtin.edu.au 
parameters of all atoms were found to vary quadratically with temperature, and were so constrained to this in the refinement, with only the coefficients of the quadratic being refined. Refinement of anisotropic displacement parameters was attempted, but was not found to be supported by the data.

The atomic positions were refined as distortion modes of a parent $\mathrm{Pm}$-3m phase as given by the ISODISTORT suite (Campbell et al. 2006). The Pnma supercell is related to its parent by the transformation matrix [[ $\left.\begin{array}{lll}1 & 0 & 1\end{array}\right],\left[\begin{array}{lll}0 & 2 & 0\end{array}\right],\left[\begin{array}{lll}-1 & 0 & 1\end{array}\right]$, with its origin at $(0,0,0)$. This structural transformation replaces the seven independent atomic position parameters of the Pnma spacegroup with seven distortion modes corresponding to two A-site cation displacements $\left(\mathrm{R}_{5}^{+}(\mathrm{A})\right.$ and $\mathrm{X}_{5}^{+}(\mathrm{A})$ ), two octahedral tilt/rotations about the $b$ and $a$ axes $\left(\mathrm{M}^{+}{ }_{3}(\mathrm{O})\right.$ and $\mathrm{R}_{4}^{+}(\mathrm{O})$ ), and three octahedral distortions $\left(\mathrm{M}_{2}^{+}(\mathrm{O}), \mathrm{R}_{5}^{+}(\mathrm{O})\right.$, and $\left.\mathrm{X}_{5}^{+}(\mathrm{O})\right)$, where $\mathrm{X}_{5}^{+}(\mathrm{O})$ corresponds to a shear along the $a$ axis.

The diffraction pattern and resultant Rietveld model for the data at $295 \mathrm{~K}$ are shown in Figure 1, with the refined structure shown in Figure 2. A summary of the structural parameters is given in Table I, showing that this material is isostructural with $\mathrm{GdFeO}_{3}$ (Geller 1956). The supplemental information contains tables all of the structural data and the values in figures $3-7$. To account for the contribution of the impurity phase, it was modelled as a set of peaks constrained by cubic $P$ $43 n$ symmetry, with changes in peak positions, intensity, and width with changes in temperature accommodated by refining a cell parameter, scale factor, and crystallite size parameter. The relative peak intensities of the impurity phase were fixed from refinement of the room-temperature model.

The orthorhombic symmetry of this perovskite can be explained as a distortion from the parent cubic perovskite phase ( $a_{p} \approx 3.76 \AA$ ) with tilts, rotations, and shears in the $\mathrm{CoO}_{6}$ octahedra and translations of the Sm atom (Glazer 1975). The orthorhombic lattice parameters are given by $a \approx$ $c \approx(\sqrt{ } 2) a_{p}$ and $b \approx 2 a_{p}$. No evidence was found for site occupancies of less than $100 \%$, with all changes in intensity and peak position able to be described by changes in atom positions, unit cell parameters, and isotropic displacement parameters. There was no evidence of preferred orientation.

The temperature dependence of the distortion modes, as it influenced the Sm position and $\mathrm{CoO}_{6}$ octahedral tilt, rotation, and shear are given in Figures 3 to 5 . The data were denoised by numerical differentiation and antidifferentiation (Chartrand 2011), with $\alpha=0.01$ for the translations and 0.05 for the octahedral distortions. Both the A-site translational distortions, $\mathrm{R}_{5}^{+}(\mathrm{A})$ and $\mathrm{X}_{5}^{+}(\mathrm{A})$, were found to be significant. Only the octahedral $\mathrm{M}_{3}^{+}(\mathrm{O}), \mathrm{R}_{4}^{+}(\mathrm{O})$, and $\mathrm{X}_{5}^{+}(\mathrm{O})$ distortions were found to be significant, with the negligible $\mathrm{M}_{2}^{+}(\mathrm{O})$ and $\mathrm{R}_{5}^{+}(\mathrm{O})$ distortions fixed at zero. The $\mathrm{R}_{4}^{+}(\mathrm{O})$ distortion is a tilting of the $\mathrm{CoO}_{6}$ octahedra about the $a$ direction, and the $\mathrm{M}^{+}{ }_{3}(\mathrm{O})$ distortion is a rotation of the octahedra about the $b$ direction. The $\mathrm{X}^{+}{ }_{5}(\mathrm{O})$ distortion is a shearing of the apical oxygen of the $\mathrm{CoO}_{6}$ octahedra in the $a$ direction. The distortion magnitudes were converted to fractional coordinates and tilt/rotation/shear angles by the following formulæ:

$$
\begin{gathered}
\operatorname{Sm} x=\frac{1}{2}+0.09399 X_{5}^{+} \\
\operatorname{Sm} z=0.09399 R_{5}^{+}
\end{gathered}
$$

a) To whom correspondence should be addressed. Electronic mail: matthew.rowles@curtin.edu.au 


$$
\begin{gathered}
\text { Oct. rot. }=\arctan \left[\frac{0.06646 R_{4}^{+} c}{\frac{1}{4} b}\right] \\
\text { Oct. tilt }=\arctan \left[\frac{a\left(-\frac{1}{4}-0.04699 M_{3}^{+}\right)}{c\left(\frac{1}{4}-0.04699 M_{3}^{+}\right)}\right] \\
\text {Oct. shear }=\arctan \left[\frac{0.09399 X_{5}^{+} a}{\frac{1}{4} b}\right]
\end{gathered}
$$

where the decimal values were given by the output of the ISODISTORT suite (Campbell et al. 2006).

The A-site translational distortions are anticorrelated, with the Sm atom moving diagonally in the $x z$ plane with changes in temperature. The octahedral tilt and rotation also tend to occur in opposite directions; as the tilt increases, the rotation decreases. The octahedral shear appears to follow the same behaviour as the octahedral tilt, but leading those changes by about $140 \mathrm{~K}$.

The temperature dependence of the unit cell parameters is shown in Figure 6. Both the instantaneous and average linear thermal expansion coefficients (TECs) are shown in Figure 7, clearly showing both their non-linearity and anisotropy. The instantaneous and average TECs were calculated as

$$
\begin{aligned}
& \mathrm{TEC}_{\text {inst. }}=\frac{1}{l} \frac{\mathrm{d} l}{\mathrm{~d} T} \\
& \mathrm{TEC}_{\mathrm{ave}}=\frac{1}{l_{0}} \frac{\Delta l}{\Delta T}
\end{aligned}
$$

where $l$ and $l_{0}$ are the current and initial cell parameters, respectively, and $\Delta$ represents the difference between the current value of the cell parameter or temperature and its initial value. The derivative was calculated numerically (Chartrand 2011). The bulk TECs calculated from the data of Tu et al. (1997) are also given for comparison. The maximum instantaneous and average TECs are given in Table II.

Overall, the unit cell expands much more in the $a$ direction than in the $b$ and $c$ directions, where the expansion is essentially equal. The peak average TEC in the $a$ direction occurs at approximately $800 \mathrm{~K}$, which is well within the operating range of intermediate-temperature solid-oxide fuel cells (Brett et al. 2008), and is much greater than any of the values given by (Sun et al. 2009). Above this temperature, the thermal expansion is still non-linear, but to a greatly reduced degree. Comparison of the average TECs to the bulk TECs of Tu et al. (1997) show that a simple average of the TECs of the three crystallographic directions can explain the bulk measurements.

The large anisotropy and non-linearity in the TECs below $900 \mathrm{~K}$ has implications for the structural integrity of polycrystalline monoliths and for joints between different solid-oxide fuel cell components, as repeated temperature cycling may induce cracking and delamination. The point of greatest orthorhombic distortion occurs at $\sim 1000 \mathrm{~K}$, where the instantaneous TECs cross over.

a) To whom correspondence should be addressed. Electronic mail: matthew.rowles@curtin.edu.au 
The maximum of the instantaneous TEC for all three axes occurs at $\sim 640 \mathrm{~K}$, showing that the gross relative changes in the average TEC for all three axes are the same.

\section{CONCLUSIONS}

The structure and thermal expansion of the perovskite $\mathrm{SmCoO}_{3}$ has been determined over the temperature range 295 to $1245 \mathrm{~K}$. The structure was found to be orthorhombic (Pnma) and isostructural with $\mathrm{GdFeO}_{3}$ over the entire temperature range. The thermal expansion is both nonlinear and anisotropic with a maximum average linear thermal expansion coefficient of 34.0(3) $\times$ $10^{-6} \mathrm{~K}^{-1}, 24.05(17) \times 10^{-6} \mathrm{~K}^{-1}$, and $24.10(18) \times 10^{-6} \mathrm{~K}^{-1}$ along the $a, b$, and $c$ axes, respectively, between 814 and $875 \mathrm{~K}$. Structural information is available in the supplemental information and select temperatures have been uploaded to the Crystallography Online Database.

\section{ACKNOWLEDGEMENTS}

This research was undertaken on the Powder Diffraction beamline at the Australian Synchrotron, Victoria, Australia.

\section{REFERENCES}

Brett, D. J., A. Atkinson, N. P. Brandon, and S. J. Skinner. (2008). "Intermediate Temperature Solid Oxide Fuel Cells." Chem. Soc. Rev. 37 (8): 1568-1578.

Bruker AXS. 2014. Topas. Ver. 5.

Campbell, B. J., H. T. Stokes, D. E. Tanner, and D. M. Hatch. (2006). "Isodisplace: A Web-Based Tool for Exploring Structural Distortions." J. Appl. Crystallogr. 39 (4): 607-614.

Chartrand, R. (2011). "Numerical Differentiation of Noisy, Nonsmooth Data." ISRN Appl. Math. 2011: 164564.

Chen, K., N. Li, N. Ai, Y. Cheng, W. D. Rickard, and S. P. Jiang. (2016). "Polarization-Induced Interface and $\mathrm{Sr}$ Segregation of in Situ Assembled $\mathrm{La}_{0.6} \mathrm{Sr}_{0.4} \mathrm{Co}_{0.2} \mathrm{Fe}_{0.8} \mathrm{O}_{3-\delta}$ Electrodes on $\mathrm{Y}_{2} \mathrm{O}_{3}-\mathrm{ZrO}_{2}$ Electrolyte of Solid Oxide Fuel Cells." ACS Applied Materials and Interfaces 8 (46): 3172931737.

Coelho, A. A. (2003). "Indexing of Powder Diffraction Patterns by Iterative Use of Singular Value Decomposition." J. Appl. Crystallogr. 36 (1): 86-95.

Dong, F., D. Chen, R. Ran, H. Park, C. Kwak, and Z. Shao. (2012). "A Comparative Study of $\mathrm{Sm}_{0.5} \mathrm{Sr}_{0.5} \mathrm{MO}_{3-\delta}(\mathrm{M}=\mathrm{Co}$ and $\mathrm{Mn})$ as Oxygen Reduction Electrodes for Solid Oxide Fuel Cells." Int. J. Hydrogen Energy 37 (5): 4377-4387.

Fukunaga, H., M. Koyama, N. Takahashi, C. Wen, and K. Yamada. (2000). "Reaction Model of Dense $\mathrm{Sm}_{0.5} \mathrm{Sr}_{0.5} \mathrm{CoO}_{3}$ as SOFC Cathode." Solid State Ionics 132 (3-4): 279-285.

Geller, S. (1956). "Crystal Structure of Gadolinium Orthoferrite, $\mathrm{GdFeO}_{3} . "$ The Journal of Chemical Physics 24 (6): 1236.

Glazer, A. M. (1975). "Simple Ways of Determining Perovskite Structures." Acta Crystallogr. A 31 (6): 756-762.

Hahn, T., ed. (1995). International Tables for Crystallography (Kluwer Academic Publishers, Dordrecht, The Netherlands), 4th ed.

Ishihara, T., M. Honda, T. Shibayama, H. Minami, H. Nishiguchi, and Y. Takita. (1998). "Intermediate Temperature Solid Oxide Fuel Cells Using a New $\mathrm{LaGaO}_{3}$ Based Oxide Ion Conductor." $J$. Electrochem. Soc. 145 (9): 3177.

Kharko, O. V., L. O. Vasylechko, S. B. Ubizskii, A. Pashuk, and Y. Prots. (2014). "Structural Behaviour of Continuous Solid Solution SmCo ${ }_{1-\mathrm{x}} \mathrm{Fe}_{\mathrm{x}} \mathrm{O}$." Funct. Mater. 21 (2): 226-232.

Pérez-Cacho, J., J. Blasco, J. García, and R. Sanchez. (2000). "Relationships between Structure and Physical Properties in $\mathrm{SmNi}_{1-\mathrm{x}} \mathrm{Co}_{\mathrm{x}} \mathrm{O}_{3}$." J. Solid State Chem. 150 (1): 145-153.

a) To whom correspondence should be addressed. Electronic mail: matthew.rowles@curtin.edu.au 
Rietveld, H. M. (1969). "A Profile Refinement Method for Nuclear and Magnetic Structures." J. Appl. Crystallogr. 2 (2): 65-71.

Rowles, M. R. (2012). "CONVAS2: A Program for the Merging of Diffraction Data." Powder Diffr. 25 (03): 297-301.

Scarlett, N. V. Y., M. R. Rowles, K. S. Wallwork, and I. C. Madsen. (2011). "Sample-Displacement Correction for Whole-Pattern Profile Fitting of Powder Diffraction Data Collected in Capillary Geometry." J. Appl. Crystallogr. 44 (1): 60-64.

Schmitt, B., C. Brönnimann, E. F. Eikenberry, F. Gozzo, C. Hörmann, R. Horisberger, and B. Patterson. (2003). "Mythen Detector System." Nucl. Instrum. Methods Phys. Res., Sect. A 501 (1): 267-272.

Shao, Z., W. Zhou, and Z. Zhu. (2012). "Advanced Synthesis of Materials for Intermediate-Temperature Solid Oxide Fuel Cells." Prog. Mater Sci. 57 (4): 804-874.

Stinton, G. W., and J. S. Evans. (2007). "Parametric Rietveld Refinement." J. Appl. Crystallogr. 40 (1): 8795.

Sun, C., R. Hui, and J. Roller. (2009). "Cathode Materials for Solid Oxide Fuel Cells: A Review." J. Solid State Electrochem. 14 (7): 1125-1144.

Taylor, D. (1984). "Thermal Expansion Data: III. Sesquioxides, $\mathrm{M}_{2} \mathrm{O}_{3}$, with the Corundum and the a-, Band C- $\mathrm{M}_{2} \mathrm{O}_{3}$ Structures." British Ceramic Transactions Journal 83 (4): 92-98.

Tu, H., Y. Takeda, N. Imanishi, and O. Yamamoto. (1997). " $\mathrm{Ln}_{1-\mathrm{x}} \mathrm{Sr}_{\mathrm{x}} \mathrm{CoO}_{3}(\mathrm{Ln}=\mathrm{Sm}$, Dy) for the Electrode of Solid Oxide Fuel Cells." Solid State Ionics 100 (3-4): 283-288.

Wallwork, K. S., B. J. Kennedy, and D. Wang. (2007). "The High Resolution Powder Diffraction Beamline for the Australian Synchrotron." AIP Conf. Proc. 879: 879-882.

Wang, J. X., Y. K. Tao, J. Shao, and W. G. Wang. (2009). "Synthesis and Properties of $\left(\mathrm{La}_{0.75} \mathrm{Sr}_{0.25}\right)_{0.95} \mathrm{MnO}_{3 \pm \delta}$ Nano-Powder Prepared Via Pechini Route." J. Power Sources 186 (2): 344-348.

Xia, C., W. Rauch, F. Chen, and M. Liu. (2002). "Sm ${ }_{0.5} \mathrm{Sr}_{0.5} \mathrm{CoO}_{3}$ Cathodes for Low-Temperature SOFCs." Solid State Ionics 149 (1-2): 11-19.

Yang, L., C. Zuo, S. Wang, Z. Cheng, and M. Liu. (2008). "A Novel Composite Cathode for LowTemperature SOFCs Based on Oxide Proton Conductors." Adv. Mater. 20 (17): 3280-3283.

a) To whom correspondence should be addressed. Electronic mail: matthew.rowles@curtin.edu.au 


\section{Figure captions}

FIG. 1. Diffraction data and Rietveld model of $\mathrm{SmCoO}_{3}$ at $295 \mathrm{~K}$. Data was collected over the range 3 to $83^{\circ} 2 \theta$.

FIG. 2. The structure of $\mathrm{SmCoO}_{3}$ at $295 \mathrm{~K}$. The images clearly show the tilt and rotation of the $\mathrm{CoO}_{6}$ corner-sharing octahedra, as well as the offset of the Sm atoms from the face of the unit cell. Distortion of the octahedra is minimal.

FIG. 3. Sm fractional $x$ and $z$ coordinates as a function of temperature. The $\mathrm{R}_{5}^{+}$and $\mathrm{X}^{+}$ distortions correspond to translations of the Sm atom in the $z$ and $x$ directions, respectively. The solid line is the denoised data. Error bars are given for every third data point and represent one estimated standard deviation.

FIG. 4. $\mathrm{CoO}_{6}$ octahedral tilt and rotation distortion as a function of temperature. The solid line is the denoised data. Error bars are given for every third data point and represent one estimated standard deviation.

FIG. 5. $\mathrm{CoO}_{6}$ octahedral shear distortion as a function of temperature. The solid line is the denoised data. Error bars are given for every third data point and represent one estimated standard deviation.

FIG. 6. Unit cell parameters as a function of temperature. The non-linear expansion behaviour of all three axes is clear. The error bars are smaller than the data points. $b^{\prime}=b / \sqrt{2}$.

Fig. 7. Instantaneous (I) and average (A) linear thermal expansion coefficients (TECs) for the three cell parameters showing the expansion anisotropy. The open circles are bulk TECs calculated from the results of Tu et al. (1997). The point of maximum orthorhombic distortion occurs at the high temperature crossover of the instantaneous coefficients at $1000 \mathrm{~K}$.

a) To whom correspondence should be addressed. Electronic mail: matthew.rowles@curtin.edu.au 


\section{Tables}

Table I. Crystal data for $\mathrm{SmCoO}_{3}$ at select temperatures. Space group Pnma, $\mathrm{Z}=4$. Molar mass $=$ 257:29 $\mathrm{g} \mathrm{mol}^{-1}$. By space group symmetry, Sm $y \&$ O2 $y=1 / 4$, and Co $x ; y ; z=0 ; 0 ; 0$. The structure data for the remaining temperatures is available in the supplemental information

\begin{tabular}{|l|l|l|l|}
\hline & $295 \mathrm{~K}$ & $830 \mathrm{~K}$ & $1245 \mathrm{~K}$ \\
\hline $\mathrm{a}(\AA)$ & $5.362267(12)$ & $5.459411(10)$ & $5.505157(13)$ \\
\hline $\mathrm{b}(\AA)$ & $7.510380(14)$ & $7.606208(12)$ & $7.67164(2)$ \\
\hline c $(\AA)$ & $5.292981(11)$ & $5.360822(9)$ & $5.405593(17)$ \\
\hline Volume $\left(\AA^{3}\right)$ & $213.1624(8)$ & $222.6103(7)$ & $228.2975(11)$ \\
\hline Density $\left(\mathrm{g} \mathrm{cm}^{-3}\right)$ & $8.01722(3)$ & $7.67696(2)$ & $7.48572(4)$ \\
\hline Sm $x$ & $0.54748(5)$ & $0.54723(7)$ & $0.54586(6)$ \\
\hline Sm $z$ & $-0.00970(5)$ & $-0.00937(7)$ & $-0.00881(10)$ \\
\hline O1 $x$ & $0.2919(5)$ & $0.2938(5)$ & $0.2932(6)$ \\
\hline O1 $y$ & $0.0414(3)$ & $0.0406(3)$ & $0.0410(4)$ \\
\hline O1 $z$ & $0.7919(5)$ & $0.7938(5)$ & $0.7932(6)$ \\
\hline O2 $x$ & $-0.0162(1)$ & $-0.0173(1)$ & $-0.0135(8)$ \\
\hline O2 $z$ & $0.0828(6)$ & $0.0812(5)$ & $0.0820(8)$ \\
\hline Sm B $\left(\AA^{2}\right)$ & $0.7119(5)$ & $1.7016(8)$ & $2.4282(15)$ \\
\hline Co B $\left(\AA^{2}\right)$ & $0.5333(13)$ & $1.0966(18)$ & $1.542(3)$ \\
\hline O1 B $\left(\AA^{2}\right)$ & $0.526(5)$ & $1.822(5)$ & $2.838(7)$ \\
\hline O2 B $\left(\AA^{2}\right)$ & $0.835(8)$ & $1.937(9)$ & $2.578(13)$ \\
\hline
\end{tabular}

Table II. Maximum instantaneous and average thermal expansion coefficients and their respective temperatures and crystallographic direction. The uncertainties incorporate a coverage factor of 2 and the uncertainties were estimated from the magnitudes of the average uncertainties.

\begin{tabular}{|l|l|l|l|l|}
\hline Axis & $\begin{array}{l}\text { Instantaneous } \\
\left(\mathrm{x} 10^{-6} \mathrm{~K}^{-1}\right)\end{array}$ & $\begin{array}{l}\text { Temperature } \\
(\mathrm{K})\end{array}$ & $\begin{array}{l}\text { Average } \\
\left(\mathrm{x} 10^{-6} \mathrm{~K}^{-1}\right)\end{array}$ & $\begin{array}{l}\text { Temperature } \\
(\mathrm{K})\end{array}$ \\
\hline a & $54.5(6)$ & 640 & $34.0(3)$ & 814 \\
\hline $\mathrm{b}$ & $33.5(4)$ & 656 & $24.05(17)$ & 875 \\
\hline c & $33.1(4)$ & 640 & $24.10(18)$ & 845 \\
\hline
\end{tabular}

a) To whom correspondence should be addressed. Electronic mail: matthew.rowles@curtin.edu.au 


\section{Technical Article}

\section{Temperature-dependent structural behaviour of samarium cobalt oxide}

Jiang ${ }^{1}$

Matthew R. Rowles ${ }^{\mathrm{a}), 1}$, Cheng Wang ${ }^{1}$, Kongfa Chen ${ }^{1}$, $\mathrm{Na} \mathrm{Li}^{1,2}$, Shuai $\mathrm{He}^{1}$ and San-Ping

${ }^{1}$ Fuels and Energy Technology Institute, Curtin University, GPO Box U1987, Perth WA, Australia, 6185

${ }^{2}$ College of Science, Heilongjiang University of Science and Technology, Harbin 150022, China

Supplementary information

a) To whom correspondence should be addressed. Electronic mail:

matthew.rowles@curtin.edu.au 
Table SI. Lattice parameters, volume, and density for $\mathrm{SmCoO}_{3}$ at all temperatures. Space group Pnma, $\mathrm{Z}=4$. Molar mass $=257.29 \mathrm{~g} \mathrm{~mol}^{-1}$.

\begin{tabular}{|c|c|c|c|c|c|}
\hline Temp. (K) & a $(\AA)$ & $\mathrm{b}(\AA)$ & с $(\AA)$ & vol. $\left(\AA^{3}\right)$ & dens. $\left(\mathrm{g} / \mathrm{cm}^{3}\right)$ \\
\hline 295 & $5.362267(12)$ & $7.510380(14)$ & $5.292981(11)$ & 213.1624(8) & $8.01722(3)$ \\
\hline 315 & $5.362750(13)$ & $7.511722(13)$ & $5.293965(12)$ & 213.2594(8) & $8.01358(3)$ \\
\hline 332 & $5.363407(13)$ & $7.513198(14)$ & $5.295079(10)$ & 213.3723(8) & $8.00934(3)$ \\
\hline 350 & $5.364257(13)$ & $7.514790(13)$ & $5.296299(12)$ & 213.5005(8) & $8.00453(3)$ \\
\hline 368 & $5.365335(14)$ & $7.516596(13)$ & $5.297619(10)$ & $213.6480(8)$ & $7.99900(3)$ \\
\hline 386 & $5.366736(14)$ & $7.518513(14)$ & $5.299061(11)$ & 213.8165(8) & $7.99270(3)$ \\
\hline 403 & $5.368338(14)$ & $7.520568(15)$ & $5.300603(11)$ & $214.0010(8)$ & $7.98581(3)$ \\
\hline 421 & $5.370148(15)$ & $7.522760(15)$ & $5.302248(11)$ & $214.2020(8)$ & 7.97831(3) \\
\hline 439 & $5.372432(15)$ & $7.525280(15)$ & $5.304110(11)$ & 214.4401(9) & $7.96945(3)$ \\
\hline 455 & $5.374787(14)$ & 7.527772(15) & $5.305972(10)$ & 214.6805(8) & $7.96053(3)$ \\
\hline 473 & $5.377486(16)$ & $7.530458(13)$ & $5.307942(11)$ & $214.9448(9)$ & 7.95074(3) \\
\hline 490 & $5.380379(15)$ & $7.533347(13)$ & $5.310039(10)$ & $215.2279(8)$ & $7.94029(3)$ \\
\hline 507 & $5.383488(15)$ & $7.536317(14)$ & $5.312201(12)$ & 215.5249(9) & $7.92934(3)$ \\
\hline 524 & $5.386789(15)$ & $7.539428(14)$ & 5.314421(11) & $215.8362(8)$ & 7.91791(3) \\
\hline 541 & $5.390087(18)$ & $7.542522(13)$ & $5.316657(10)$ & $216.1479(9)$ & $7.90649(3)$ \\
\hline 558 & $5.393918(15)$ & $7.545908(13)$ & $5.319116(11)$ & 216.4987(8) & $7.89368(3)$ \\
\hline 575 & $5.397829(16)$ & $7.549416(14)$ & $5.321627(11)$ & 216.8587(9) & $7.88057(3)$ \\
\hline 591 & 5.401923(16) & $7.553011(14)$ & $5.324188(11)$ & $217.2310(9)$ & $7.86707(3)$ \\
\hline 608 & $5.406215(15)$ & $7.556727(14)$ & $5.326876(11)$ & $217.6204(8)$ & $7.85299(3)$ \\
\hline 624 & $5.410817(13)$ & $7.560650(13)$ & $5.329638(11)$ & $218.0317(8)$ & 7.83818(3) \\
\hline 640 & $5.415360(13)$ & $7.564535(13)$ & $5.332335(11)$ & 218.4374(8) & 7.82362(3) \\
\hline 656 & $5.420069(13)$ & 7.568523(13) & $5.335119(12)$ & $218.8568(8)$ & $7.80863(3)$ \\
\hline 673 & $5.424780(13)$ & $7.572557(13)$ & $5.337906(11)$ & 219.2783(8) & 7.79362(3) \\
\hline 689 & $5.429538(12)$ & $7.576698(12)$ & $5.340777(11)$ & $219.7087(8)$ & 7.77835(3) \\
\hline 705 & $5.433665(11)$ & $7.580402(10)$ & $5.343311(11)$ & $220.0876(7)$ & 7.76496(2) \\
\hline 721 & $5.437758(13)$ & $7.584137(13)$ & $5.345880(10)$ & $220.4679(8)$ & 7.75156(3) \\
\hline 737 & $5.441235(12)$ & $7.587412(11)$ & $5.348113(10)$ & $220.7963(7)$ & 7.74003(2) \\
\hline 752 & $5.444670(11)$ & $7.590678(13)$ & $5.350340(10)$ & $221.1228(7)$ & $7.72861(2)$ \\
\hline 768 & $5.447469(12)$ & 7.593431(13) & $5.352210(10)$ & 221.3941(7) & 7.71914(3) \\
\hline 783 & $5.450935(13)$ & $7.596998(13)$ & $5.354632(10)$ & $221.7393(8)$ & $7.70712(3)$ \\
\hline 799 & $5.453631(11)$ & 7.599788(14) & $5.356509(11)$ & $222.0082(8)$ & 7.69778(3) \\
\hline 814 & $5.456938(10)$ & $7.603417(12)$ & $5.358958(10)$ & $222.3505(7)$ & 7.68593(2) \\
\hline 830 & $5.459411(10)$ & 7.606208(12) & $5.360822(9)$ & $222.6103(7)$ & 7.67696(2) \\
\hline 845 & 5.462339(9) & $7.609539(12)$ & $5.363080(9)$ & 222.9211(6) & 7.66626(2) \\
\hline 860 & 5.464453(9) & 7.611987(13) & 5.364712(9) & $223.1470(7)$ & $7.65850(2)$ \\
\hline 875 & $5.467000(9)$ & $7.615058(13)$ & $5.366799(10)$ & $223.4280(7)$ & $7.64887(2)$ \\
\hline 889 & $5.469117(9)$ & 7.617661(15) & $5.368576(10)$ & $223.6650(7)$ & $7.64076(2)$ \\
\hline
\end{tabular}

a) To whom correspondence should be addressed. Electronic mail:

matthew.rowles@curtin.edu.au 


\begin{tabular}{|l|l|l|l|l|l|}
\hline 904 & $5.471187(10)$ & $7.620258(13)$ & $5.370311(9)$ & $223.8982(7)$ & $7.63280(2)$ \\
\hline 919 & $5.473089(10)$ & $7.622650(14)$ & $5.371919(10)$ & $224.1135(7)$ & $7.62547(2)$ \\
\hline 934 & $5.474805(10)$ & $7.624891(14)$ & $5.373453(11)$ & $224.3137(8)$ & $7.61866(3)$ \\
\hline 948 & $5.477068(10)$ & $7.627873(14)$ & $5.375452(10)$ & $224.5776(7)$ & $7.60971(2)$ \\
\hline 963 & $5.478677(9)$ & $7.630062(14)$ & $5.376942(11)$ & $224.7704(7)$ & $7.60318(2)$ \\
\hline 977 & $5.480447(9)$ & $7.632521(13)$ & $5.378571(10)$ & $224.9836(7)$ & $7.59598(2)$ \\
\hline 991 & $5.482013(10)$ & $7.634692(15)$ & $5.380069(11)$ & $225.1746(8)$ & $7.58954(3)$ \\
\hline 1005 & $5.483778(9)$ & $7.637130(15)$ & $5.381728(11)$ & $225.3885(8)$ & $7.58233(3)$ \\
\hline 1019 & $5.485170(9)$ & $7.639168(15)$ & $5.383113(11)$ & $225.5640(7)$ & $7.57644(3)$ \\
\hline 1034 & $5.486466(10)$ & $7.641077(17)$ & $5.384421(12)$ & $225.7284(8)$ & $7.57092(3)$ \\
\hline 1048 & $5.487968(9)$ & $7.643280(15)$ & $5.385927(10)$ & $225.9185(7)$ & $7.56455(2)$ \\
\hline 1061 & $5.489331(9)$ & $7.645302(17)$ & $5.387313(10)$ & $226.0926(8)$ & $7.55872(3)$ \\
\hline 1076 & $5.490659(10)$ & $7.647310(18)$ & $5.388670(12)$ & $226.2636(8)$ & $7.55301(3)$ \\
\hline 1089 & $5.491902(11)$ & $7.649258(19)$ & $5.390011(13)$ & $226.4288(9)$ & $7.54750(3)$ \\
\hline 1103 & $5.493166(11)$ & $7.651246(18)$ & $5.391391(12)$ & $226.5978(9)$ & $7.54187(3)$ \\
\hline 1116 & $5.494448(9)$ & $7.653327(18)$ & $5.392807(12)$ & $226.7718(8)$ & $7.53608(3)$ \\
\hline 1130 & $5.495533(11)$ & $7.655029(18)$ & $5.394009(12)$ & $226.9177(9)$ & $7.53124(3)$ \\
\hline 1142 & $5.496933(11)$ & $7.657297(19)$ & $5.395592(12)$ & $227.1093(9)$ & $7.52488(3)$ \\
\hline 1156 & $5.497982(11)$ & $7.659079(20)$ & $5.396800(12)$ & $227.2564(9)$ & $7.52001(3)$ \\
\hline 1169 & $5.499181(12)$ & $7.661091(20)$ & $5.398208(14)$ & $227.4250(10)$ & $7.51444(3)$ \\
\hline 1181 & $5.500281(11)$ & $7.662970(20)$ & $5.399508(15)$ & $227.5811(10)$ & $7.50928(3)$ \\
\hline 1195 & $5.501171(13)$ & $7.664560(21)$ & $5.400625(14)$ & $227.7123(10)$ & $7.50496(3)$ \\
\hline 1207 & $5.502118(12)$ & $7.666162(24)$ & $5.401748(15)$ & $227.8464(11)$ & $7.50054(4)$ \\
\hline 1220 & $5.503401(14)$ & $7.668478(22)$ & $5.403385(15)$ & $228.0375(11)$ & $7.49425(4)$ \\
\hline 1233 & $5.504310(12)$ & $7.670106(23)$ & $5.404561(14)$ & $228.1732(10)$ & $7.48980(3)$ \\
\hline 1245 & $5.505157(13)$ & $7.671638(24)$ & $5.405593(17)$ & $228.2975(11)$ & $7.48572(4)$ \\
\hline
\end{tabular}

a) To whom correspondence should be addressed. Electronic mail: matthew.rowles@curtin.edu.au 
Table SII. Atom positions for $\mathrm{SmCoO}_{3}$ at all temperatures. Space group Pnma, $\mathrm{Z}=4$. Molar mass $=257.29 \mathrm{~g} \mathrm{~mol}^{-1}$. By space group symmetry, Sm y \& O2 $y=1 / 4$, and Co $x ; y ; z=0 ; 0 ; 0$.

\begin{tabular}{|c|c|c|c|c|c|c|c|}
\hline Temp. (K) & Sm_x & Sm_z & O1_x & O1_y & O1_z & O2_x & O2_z \\
\hline 295 & $0.54748(5)$ & $-0.00970(5)$ & $0.2919(5)$ & $0.0414(3)$ & $0.7919(5)$ & $-0.0162(10)$ & $0.0828(6)$ \\
\hline 315 & $0.54736(6)$ & $-0.00957(6)$ & $0.2921(5)$ & $0.0412(3)$ & $0.7921(5)$ & $-0.0165(9)$ & $0.0825(5)$ \\
\hline 332 & $0.54722(6)$ & $-0.00958(6)$ & $0.2919(5)$ & $0.0414(3)$ & 0.7919(5) & $-0.0172(9)$ & $0.0828(5)$ \\
\hline 350 & $0.54709(6)$ & $-0.00950(6)$ & $0.2919(5)$ & $0.0416(3)$ & $0.7919(5)$ & $-0.0178(10)$ & $0.0831(5)$ \\
\hline 368 & $0.54697(6)$ & $-0.00944(5)$ & $0.2919(5)$ & $0.0413(3)$ & $0.7919(5)$ & $-0.0169(10)$ & $0.0826(6)$ \\
\hline 386 & $0.54689(6)$ & $-0.00937(5)$ & $0.2918(5)$ & $0.0415(3)$ & $0.7918(5)$ & $-0.0168(10)$ & $0.0829(5)$ \\
\hline 403 & $0.54673(6)$ & $-0.00932(6)$ & $0.2918(5)$ & $0.0413(3)$ & $0.7918(5)$ & $-0.0170(10)$ & $0.0826(5)$ \\
\hline 421 & $0.54671(6)$ & $-0.00917(5)$ & $0.2919(5)$ & $0.0415(3)$ & $0.7919(5)$ & $-0.0166(11)$ & $0.0830(5)$ \\
\hline 439 & $0.54662(7)$ & $-0.00911(6)$ & $0.2922(5)$ & $0.0414(3)$ & $0.7922(5)$ & $-0.0169(11)$ & $0.0828(6)$ \\
\hline 455 & $0.54656(6)$ & $-0.00915(6)$ & $0.2921(6)$ & $0.0414(3)$ & $0.7921(6)$ & $-0.0168(11)$ & $0.0828(6)$ \\
\hline 473 & $0.54651(6)$ & $-0.00900(6)$ & $0.2922(5)$ & $0.0411(3)$ & $0.7922(5)$ & $-0.0178(10)$ & $0.0822(5)$ \\
\hline 490 & $0.54652(6)$ & $-0.00904(6)$ & $0.2922(5)$ & $0.0413(3)$ & $0.7922(5)$ & $-0.0178(11)$ & $0.0826(6)$ \\
\hline 507 & $0.54650(7)$ & $-0.00891(6)$ & $0.2921(5)$ & $0.0415(3)$ & $0.7921(5)$ & $-0.0183(11)$ & $0.0830(6)$ \\
\hline 524 & $0.54655(6)$ & $-0.00890(6)$ & $0.2920(5)$ & $0.0414(3)$ & $0.7920(5)$ & $-0.0190(10)$ & $0.0828(5)$ \\
\hline 541 & $0.54654(7)$ & $-0.00897(7)$ & $0.2917(6)$ & $0.0412(3)$ & $0.7917(6)$ & $-0.0196(12)$ & $0.0824(5)$ \\
\hline 558 & $0.54653(7)$ & $-0.00894(7)$ & $0.2916(5)$ & $0.0415(3)$ & $0.7916(5)$ & $-0.0196(11)$ & $0.0829(6)$ \\
\hline 575 & $0.54650(7)$ & $-0.00901(7)$ & $0.2921(5)$ & $0.0413(3)$ & $0.7921(5)$ & $-0.0188(11)$ & $0.0826(6)$ \\
\hline 591 & $0.54644(7)$ & $-0.00909(7)$ & $0.2922(6)$ & $0.0411(3)$ & $0.7922(6)$ & $-0.0165(13)$ & $0.0823(6)$ \\
\hline 608 & $0.54666(7)$ & $-0.00914(6)$ & $0.2920(6)$ & $0.0415(3)$ & $0.7920(6)$ & $-0.0184(12)$ & $0.0831(6)$ \\
\hline 624 & $0.54670(8)$ & $-0.00915(7)$ & $0.2922(5)$ & $0.0413(3)$ & $0.7922(5)$ & $-0.0179(12)$ & $0.0826(6)$ \\
\hline 640 & $0.54668(8)$ & $-0.00912(6)$ & $0.2926(5)$ & $0.0415(3)$ & $0.7926(5)$ & $-0.0163(11)$ & $0.0830(6)$ \\
\hline 656 & $0.54684(7)$ & $-0.00912(7)$ & $0.2931(5)$ & $0.0419(3)$ & $0.7931(5)$ & $-0.0164(11)$ & $0.0837(6)$ \\
\hline 673 & $0.54696(7)$ & $-0.00910(7)$ & $0.2931(6)$ & $0.0418(3)$ & $0.7931(6)$ & $-0.0149(13)$ & $0.0837(6)$ \\
\hline 689 & $0.54707(7)$ & $-0.00921(7)$ & $0.2932(5)$ & $0.0416(3)$ & $0.7932(5)$ & $-0.0130(12)$ & $0.0833(6)$ \\
\hline 705 & $0.54712(7)$ & $-0.00913(7)$ & $0.2938(5)$ & $0.0414(3)$ & $0.7938(5)$ & $-0.0136(12)$ & $0.0828(7)$ \\
\hline 721 & $0.54725(7)$ & $-0.00917(7)$ & $0.2934(6)$ & $0.0416(3)$ & $0.7934(6)$ & $-0.0149(14)$ & $0.0832(6)$ \\
\hline 737 & $0.54712(7)$ & $-0.00920(6)$ & $0.2936(5)$ & $0.0412(3)$ & $0.7936(5)$ & $-0.0163(12)$ & $0.0824(6)$ \\
\hline 752 & $0.54724(8)$ & $-0.00934(7)$ & $0.2945(5)$ & $0.0412(3)$ & $0.7945(5)$ & $-0.0158(12)$ & $0.0823(5)$ \\
\hline 768 & $0.54733(7)$ & $-0.00922(7)$ & $0.2935(5)$ & $0.0415(3)$ & $0.7935(5)$ & $-0.0155(13)$ & $0.0830(6)$ \\
\hline 783 & $0.54720(7)$ & $-0.00937(6)$ & $0.2941(5)$ & $0.0411(3)$ & $0.7941(5)$ & $-0.0162(11)$ & $0.0821(5)$ \\
\hline 799 & $0.54730(7)$ & $-0.00929(7)$ & $0.2938(5)$ & $0.0408(3)$ & $0.7938(5)$ & $-0.0169(11)$ & $0.0817(5)$ \\
\hline 814 & $0.54733(7)$ & $-0.00943(7)$ & $0.2940(4)$ & $0.0407(2)$ & $0.7940(4)$ & $-0.0166(11)$ & $0.0814(5)$ \\
\hline 830 & $0.54723(7)$ & $-0.00937(7)$ & $0.2938(5)$ & $0.0406(3)$ & $0.7938(5)$ & $-0.0173(10)$ & $0.0812(5)$ \\
\hline 845 & $0.54737(7)$ & $-0.00948(6)$ & $0.2941(4)$ & $0.0407(3)$ & $0.7941(4)$ & $-0.0167(10)$ & $0.0813(5)$ \\
\hline 860 & $0.54723(6)$ & $-0.00929(6)$ & $0.2939(5)$ & $0.0407(3)$ & $0.7939(5)$ & $-0.0164(10)$ & $0.0813(5)$ \\
\hline 875 & $0.54734(7)$ & $-0.00937(6)$ & $0.2940(5)$ & $0.0407(2)$ & $0.7940(5)$ & $-0.0156(10)$ & $0.0814(5)$ \\
\hline 889 & $0.54727(6)$ & $-0.00957(6)$ & $0.2940(4)$ & $0.0406(3)$ & $0.7940(4)$ & $-0.0166(9)$ & $0.0812(5)$ \\
\hline
\end{tabular}

a) To whom correspondence should be addressed. Electronic mail: matthew.rowles@curtin.edu.au 


\begin{tabular}{|c|c|c|c|c|c|c|c|}
\hline 904 & $0.54720(6)$ & $-0.00931(7)$ & $0.2936(5)$ & $0.0410(2)$ & $0.7936(5)$ & $-0.0161(10)$ & $0.0819(4)$ \\
\hline 919 & $0.54727(6)$ & $-0.00939(7)$ & $0.2939(4)$ & $0.0408(3)$ & $0.7939(4)$ & $-0.0154(9)$ & $0.0817(5)$ \\
\hline 934 & $0.54728(6)$ & $-0.00936(7)$ & $0.2934(4)$ & $0.0408(3)$ & $0.7934(4)$ & $-0.0170(10)$ & $0.0816(6)$ \\
\hline 948 & $0.54713(6)$ & $-0.00937(7)$ & $0.2938(4)$ & $0.0407(2)$ & $0.7938(4)$ & $-0.0162(9)$ & $0.0815(5)$ \\
\hline 963 & $0.54715(6)$ & $-0.00932(7)$ & $0.2936(5)$ & $0.0410(3)$ & $0.7936(5)$ & $-0.0162(9)$ & $0.0820(5)$ \\
\hline 977 & $0.54702(6)$ & $-0.00920(7)$ & $0.2933(4)$ & $0.0411(3)$ & $0.7933(4)$ & $-0.0162(9)$ & $0.0822(5)$ \\
\hline 991 & $0.54702(6)$ & $-0.00913(7)$ & $0.2937(4)$ & $0.0410(2)$ & $0.7937(4)$ & $-0.0161(9)$ & $0.0819(5)$ \\
\hline 1005 & $0.54698(5)$ & $-0.00908(7)$ & $0.2927(5)$ & $0.0410(3)$ & $0.7927(5)$ & $-0.0154(8)$ & $0.0821(5)$ \\
\hline 1019 & $0.54698(6)$ & $-0.00922(7)$ & $0.2931(5)$ & $0.0410(3)$ & $0.7931(5)$ & $-0.0147(8)$ & $0.0819(5)$ \\
\hline 1034 & $0.54692(6)$ & $-0.00907(7)$ & $0.2930(5)$ & $0.0412(3)$ & $0.7930(5)$ & $-0.0154(8)$ & $0.0824(5)$ \\
\hline 1048 & $0.54687(6)$ & $-0.00913(7)$ & $0.2933(5)$ & $0.0412(3)$ & $0.7933(5)$ & $-0.0157(7)$ & $0.0824(5)$ \\
\hline 1061 & $0.54680(6)$ & $-0.00910(8)$ & $0.2927(4)$ & $0.0412(3)$ & $0.7927(4)$ & $-0.0153(8)$ & $0.0824(6)$ \\
\hline 1076 & $0.54681(5)$ & $-0.00908(7)$ & $0.2930(5)$ & $0.0412(3)$ & $0.7930(5)$ & $-0.0149(7)$ & $0.0823(6)$ \\
\hline 1089 & $0.54675(6)$ & $-0.00909(8)$ & $0.2930(4)$ & $0.0411(3)$ & $0.7930(4)$ & $-0.0141(8)$ & $0.0821(6)$ \\
\hline 1103 & $0.54666(5)$ & $-0.00914(8)$ & $0.2930(5)$ & $0.0409(3)$ & $0.7930(5)$ & $-0.0143(7)$ & $0.0819(6)$ \\
\hline 1116 & $0.54661(5)$ & $-0.00893(8)$ & $0.2929(5)$ & $0.0412(3)$ & $0.7929(5)$ & $-0.0133(8)$ & $0.0825(6)$ \\
\hline 1130 & $0.54657(6)$ & $-0.00913(7)$ & $0.2936(5)$ & $0.0410(3)$ & $0.7936(5)$ & $-0.0131(7)$ & $0.0821(6)$ \\
\hline 1142 & $0.54642(5)$ & $-0.00904(8)$ & $0.2930(5)$ & $0.0409(3)$ & $0.7930(5)$ & $-0.0135(8)$ & $0.0818(6)$ \\
\hline 1156 & $0.54640(6)$ & $-0.00905(8)$ & $0.2927(6)$ & $0.0410(3)$ & $0.7927(6)$ & $-0.0127(8)$ & $0.0819(6)$ \\
\hline 1169 & $0.54635(5)$ & $-0.00900(9)$ & $0.2930(5)$ & $0.0412(3)$ & $0.7930(5)$ & $-0.0127(7)$ & $0.0823(6)$ \\
\hline 1181 & $0.54631(5)$ & $-0.00894(9)$ & $0.2929(5)$ & $0.0411(3)$ & $0.7929(5)$ & $-0.0122(7)$ & $0.0821(6)$ \\
\hline 1195 & $0.54623(5)$ & $-0.00881(8)$ & $0.2928(5)$ & $0.0410(3)$ & $0.7928(5)$ & $-0.0137(7)$ & $0.0819(6)$ \\
\hline 1207 & $0.54611(5)$ & $\begin{array}{c}-0.00889(9) \\
\end{array}$ & $0.2927(6)$ & $0.0411(4)$ & $0.7927(6)$ & $-0.0124(8)$ & $0.0822(7)$ \\
\hline 1220 & $0.54598(6)$ & $-0.00908(9)$ & $0.2932(6)$ & $0.0407(3)$ & $0.7932(6)$ & $-0.0133(7)$ & $0.0814(7)$ \\
\hline 1233 & $0.54607(6)$ & $-0.00916(9)$ & $0.2926(6)$ & $0.0405(4)$ & $0.7926(6)$ & $-0.0129(7)$ & $0.0810(7)$ \\
\hline 1245 & $0.54586(6)$ & $-0.00881(10)$ & $0.2932(6)$ & $0.0410(4)$ & $0.7932(6)$ & $-0.0135(8)$ & $0.0820(8)$ \\
\hline
\end{tabular}

a) To whom correspondence should be addressed. Electronic mail: matthew.rowles@curtin.edu.au 
Table SIII. Isotropic thermal parameters for $\mathrm{SmCoO}_{3}$ at all temperatures.

\begin{tabular}{|c|c|c|c|c|}
\hline Temp. (K) & Sm_beq $\left(\AA^{2}\right)$ & Co_beq $\left(\AA^{2}\right)$ & O1_beq $\left(\AA^{2}\right)$ & O2_beq $\left(\AA^{2}\right)$ \\
\hline 295 & $0.7119(5)$ & $0.5333(13)$ & $0.526(5)$ & $0.835(8)$ \\
\hline 315 & $0.7488(5)$ & $0.5536(13)$ & $0.573(5)$ & $0.880(8)$ \\
\hline 332 & $0.7823(5)$ & $0.5719(13)$ & $0.615(5)$ & $0.921(8)$ \\
\hline 350 & $0.8161(5)$ & $0.5905(13)$ & $0.658(5)$ & $0.962(8)$ \\
\hline 368 & $0.8497(5)$ & 0.6091(13) & $0.701(5)$ & $1.003(8)$ \\
\hline 386 & $0.8842(5)$ & $0.6281(13)$ & $0.745(5)$ & $1.044(8)$ \\
\hline 403 & $0.9160(5)$ & $0.6458(13)$ & $0.786(5)$ & $1.082(8)$ \\
\hline 421 & $0.9500(5)$ & $0.6647(13)$ & $0.829(5)$ & $1.122(8)$ \\
\hline 439 & $0.9833(5)$ & $0.6833(13)$ & $0.872(5)$ & $1.161(8)$ \\
\hline 455 & $1.0151(5)$ & $0.7011(13)$ & $0.913(5)$ & $1.198(8)$ \\
\hline 473 & $1.0470(6)$ & $0.7190(13)$ & $0.954(5)$ & $1.235(8)$ \\
\hline 490 & 1.0798(6) & $0.7374(13)$ & $0.997(5)$ & $1.273(8)$ \\
\hline 507 & $1.1115(6)$ & $0.7553(14)$ & $1.038(5)$ & $1.309(8)$ \\
\hline 524 & 1.1434(6) & $0.7733(14)$ & $1.079(5)$ & $1.345(8)$ \\
\hline 541 & $1.1743(6)$ & $0.7908(14)$ & $1.120(5)$ & $1.380(8)$ \\
\hline 558 & $1.2063(6)$ & $0.8090(14)$ & 1.161(5) & $1.416(8)$ \\
\hline 575 & $1.2368(6)$ & $0.8263(14)$ & $1.201(5)$ & $1.450(8)$ \\
\hline 591 & $1.2679(6)$ & $0.8441(14)$ & $1.242(5)$ & $1.484(8)$ \\
\hline 608 & $1.2979(6)$ & $0.8613(14)$ & $1.282(5)$ & $1.517(8)$ \\
\hline 624 & $1.3273(6)$ & $0.8781(15)$ & $1.320(5)$ & $1.549(8)$ \\
\hline 640 & $1.3581(6)$ & $0.8959(15)$ & $1.361(5)$ & $1.582(8)$ \\
\hline 656 & $1.3873(6)$ & $0.9127(15)$ & $1.400(5)$ & $1.614(8)$ \\
\hline 673 & $1.4175(7)$ & $0.9302(15)$ & $1.440(5)$ & 1.646(8) \\
\hline 689 & $1.4462(7)$ & $0.9468(15)$ & $1.478(5)$ & $1.676(8)$ \\
\hline 705 & $1.4763(7)$ & $0.9643(16)$ & $1.518(5)$ & $1.708(8)$ \\
\hline 721 & $1.5042(7)$ & $0.9805(16)$ & $1.555(5)$ & $1.737(8)$ \\
\hline 737 & $1.5337(7)$ & $0.9978(16)$ & $1.595(5)$ & $1.768(8)$ \\
\hline 752 & $1.5615(7)$ & $1.0141(16)$ & $1.632(5)$ & $1.796(8)$ \\
\hline 768 & $1.5902(7)$ & $1.0309(16)$ & $1.671(5)$ & $1.825(8)$ \\
\hline 783 & $1.6180(7)$ & $1.0472(17)$ & $1.708(5)$ & $1.854(9)$ \\
\hline 799 & 1.6463(8) & 1.0639(17) & $1.747(5)$ & 1.882(9) \\
\hline 814 & $1.6734(8)$ & $1.0800(17)$ & $1.783(5)$ & $1.909(9)$ \\
\hline 830 & $1.7016(8)$ & $1.0966(18)$ & $1.822(5)$ & 1.937(9) \\
\hline 845 & $1.7280(8)$ & $1.1123(18)$ & $1.857(5)$ & $1.963(9)$ \\
\hline 860 & 1.7558(8) & 1.1288(18) & $1.895(6)$ & $1.990(9)$ \\
\hline 875 & 1.7819(9) & $1.1444(18)$ & 1.931(6) & $2.015(9)$ \\
\hline 889 & $1.8080(9)$ & 1.1601(19) & $1.967(6)$ & $2.040(9)$ \\
\hline 904 & 1.8348(9) & 1.1761(19) & $2.003(6)$ & $2.066(9)$ \\
\hline
\end{tabular}

a) To whom correspondence should be addressed. Electronic mail: matthew.rowles@curtin.edu.au 


\begin{tabular}{|l|l|l|l|l|}
\hline 919 & $1.8605(9)$ & $1.1916(19)$ & $2.039(6)$ & $2.090(9)$ \\
\hline 934 & $1.8869(9)$ & $1.207(2)$ & $2.075(6)$ & $2.115(9)$ \\
\hline 948 & $1.9122(10)$ & $1.223(2)$ & $2.110(6)$ & $2.139(9)$ \\
\hline 963 & $1.9383(10)$ & $1.238(2)$ & $2.146(6)$ & $2.163(10)$ \\
\hline 977 & $1.9632(10)$ & $1.254(2)$ & $2.181(6)$ & $2.186(10)$ \\
\hline 991 & $1.9877(10)$ & $1.268(2)$ & $2.215(6)$ & $2.208(10)$ \\
\hline 1005 & $2.0132(10)$ & $1.284(2)$ & $2.250(6)$ & $2.231(10)$ \\
\hline 1019 & $2.0373(11)$ & $1.299(2)$ & $2.284(6)$ & $2.253(10)$ \\
\hline 1034 & $2.0624(11)$ & $1.314(2)$ & $2.319(6)$ & $2.275(10)$ \\
\hline 1048 & $2.0874(11)$ & $1.329(2)$ & $2.354(6)$ & $2.297(10)$ \\
\hline 1061 & $2.1108(11)$ & $1.344(2)$ & $2.387(6)$ & $2.317(10)$ \\
\hline 1076 & $2.1356(12)$ & $1.359(2)$ & $2.421(6)$ & $2.339(11)$ \\
\hline 1089 & $2.1584(12)$ & $1.373(2)$ & $2.453(7)$ & $2.358(11)$ \\
\hline 1103 & $2.1828(12)$ & $1.388(2)$ & $2.488(7)$ & $2.379(11)$ \\
\hline 1116 & $2.2057(12)$ & $1.402(3)$ & $2.520(7)$ & $2.399(11)$ \\
\hline 1130 & $2.2293(13)$ & $1.417(3)$ & $2.554(7)$ & $2.418(11)$ \\
\hline 1142 & $2.2516(13)$ & $1.431(3)$ & $2.585(7)$ & $2.437(11)$ \\
\hline 1156 & $2.2750(13)$ & $1.446(3)$ & $2.619(7)$ & $2.456(11)$ \\
\hline 1169 & $2.2970(13)$ & $1.459(3)$ & $2.650(7)$ & $2.474(12)$ \\
\hline 1181 & $2.3187(14)$ & $1.473(3)$ & $2.681(7)$ & $2.492(12)$ \\
\hline 1195 & $2.3417(14)$ & $1.487(3)$ & $2.714(7)$ & $2.510(12)$ \\
\hline 1207 & $2.3628(14)$ & $1.501(3)$ & $2.744(7)$ & $2.527(12)$ \\
\hline 1220 & $2.3853(15)$ & $1.515(3)$ & $2.776(7)$ & $2.545(12)$ \\
\hline 1233 & $2.4065(15)$ & $1.528(3)$ & $2.807(7)$ & $2.561(12)$ \\
\hline 1245 & $2.4282(15)$ & $1.542(3)$ & $2.838(7)$ & $2.578(13)$ \\
\hline
\end{tabular}

a) To whom correspondence should be addressed. Electronic mail: matthew.rowles@curtin.edu.au 
Table SIV. Octahedral tilt, rotation, and shear for $\mathrm{CoO}_{6}$ at all temperatures.

\begin{tabular}{|c|c|c|c|}
\hline Temp. (K) & Tilt $\left(^{\circ}\right)$ & Rotation $\left(^{\circ}\right)$ & Shear $\left(^{\circ}\right)$ \\
\hline 295 & 13.13(8) & $9.15(7)$ & $2.65(17)$ \\
\hline 315 & 13.09(8) & $9.22(7)$ & $2.70(15)$ \\
\hline 332 & 13.13(8) & $9.16(7)$ & $2.81(14)$ \\
\hline 350 & 13.19(8) & $9.16(8)$ & 2.91(16) \\
\hline 368 & 13.11(8) & $9.16(8)$ & $2.76(16)$ \\
\hline 386 & 13.16(8) & $9.16(8)$ & $2.74(16)$ \\
\hline 403 & 13.12(8) & $9.15(7)$ & $2.77(17)$ \\
\hline 421 & 13.18(8) & $9.18(7)$ & 2.72(19) \\
\hline 439 & 13.13(9) & $9.24(8)$ & $2.77(18)$ \\
\hline 455 & 13.14(9) & $9.21(8)$ & 2.74(18) \\
\hline 473 & 13.04(8) & $9.22(8)$ & 2.91(17) \\
\hline 490 & 13.11(9) & $9.22(8)$ & 2.91(17) \\
\hline 507 & 13.17(9) & $9.19(8)$ & $2.99(18)$ \\
\hline 524 & 13.13(8) & $9.18(7)$ & $3.12(17)$ \\
\hline 541 & $13.08(8)$ & $9.10(8)$ & $3.2(2)$ \\
\hline 558 & 13.16(9) & $9.07(7)$ & $3.21(18)$ \\
\hline 575 & 13.11(9) & $9.17(8)$ & $3.09(18)$ \\
\hline 591 & 13.06(9) & $9.18(9)$ & $2.7(2)$ \\
\hline 608 & 13.18(9) & $9.13(8)$ & $3.0(2)$ \\
\hline 624 & $13.11(10)$ & $9.17(8)$ & $2.9(2)$ \\
\hline 640 & 13.18(9) & $9.25(7)$ & $2.68(19)$ \\
\hline 656 & $13.28(10)$ & $9.36(8)$ & $2.69(18)$ \\
\hline 673 & 13.27(9) & $9.35(8)$ & $2.4(2)$ \\
\hline 689 & 13.21(9) & $9.36(7)$ & $2.14(19)$ \\
\hline 705 & 13.14(10) & $9.48(7)$ & $2.2(2)$ \\
\hline 721 & $13.20(10)$ & $9.39(8)$ & $2.4(2)$ \\
\hline 737 & 13.09(8) & $9.43(7)$ & 2.68(19) \\
\hline 752 & $13.06(8)$ & $9.61(7)$ & $2.6(2)$ \\
\hline 768 & 13.16(9) & 9.39(7) & $2.5(2)$ \\
\hline 783 & $13.04(8)$ & $9.52(7)$ & $2.67(18)$ \\
\hline 799 & $12.96(8)$ & $9.46(7)$ & $2.78(18)$ \\
\hline 814 & 12.93(8) & $9.50(6)$ & $2.74(19)$ \\
\hline 830 & 12.90(8) & $9.44(7)$ & $2.84(17)$ \\
\hline 845 & 12.91(8) & $9.51(6)$ & $2.74(16)$ \\
\hline 860 & 12.91(8) & $9.47(7)$ & $2.70(17)$ \\
\hline 875 & 12.92(7) & $9.48(7)$ & $2.56(16)$ \\
\hline 889 & 12.90(8) & $9.47(6)$ & $2.72(15)$ \\
\hline 904 & $13.00(7)$ & $9.38(7)$ & $2.65(17)$ \\
\hline
\end{tabular}

a) To whom correspondence should be addressed. Electronic mail: matthew.rowles@curtin.edu.au 


\begin{tabular}{|l|l|l|l|}
\hline 919 & $12.97(8)$ & $9.45(6)$ & $2.54(16)$ \\
\hline 934 & $12.95(9)$ & $9.34(6)$ & $2.80(16)$ \\
\hline 948 & $12.94(8)$ & $9.42(6)$ & $2.66(14)$ \\
\hline 963 & $13.02(8)$ & $9.40(7)$ & $2.66(15)$ \\
\hline 977 & $13.04(8)$ & $9.33(6)$ & $2.67(15)$ \\
\hline 991 & $13.00(7)$ & $9.41(6)$ & $2.65(14)$ \\
\hline 1005 & $13.03(8)$ & $9.19(7)$ & $2.54(14)$ \\
\hline 1019 & $13.00(8)$ & $9.26(7)$ & $2.41(14)$ \\
\hline 1034 & $13.08(8)$ & $9.26(7)$ & $2.53(13)$ \\
\hline 1048 & $13.08(8)$ & $9.31(7)$ & $2.58(11)$ \\
\hline 1061 & $13.07(9)$ & $9.19(6)$ & $2.51(13)$ \\
\hline 1076 & $13.07(9)$ & $9.25(7)$ & $2.46(12)$ \\
\hline 1089 & $13.03(9)$ & $9.24(6)$ & $2.32(13)$ \\
\hline 1103 & $13.00(9)$ & $9.24(7)$ & $2.36(12)$ \\
\hline 1116 & $13.09(10)$ & $9.24(8)$ & $2.19(14)$ \\
\hline 1130 & $13.02(9)$ & $9.39(7)$ & $2.16(12)$ \\
\hline 1142 & $12.98(10)$ & $9.26(7)$ & $2.23(13)$ \\
\hline 1156 & $13.01(10)$ & $9.19(8)$ & $2.09(13)$ \\
\hline 1169 & $13.06(10)$ & $9.26(8)$ & $2.09(12)$ \\
\hline 1181 & $13.03(10)$ & $9.23(7)$ & $2.00(12)$ \\
\hline 1195 & $13.01(9)$ & $9.22(8)$ & $2.25(12)$ \\
\hline 1207 & $13.04(11)$ & $9.20(9)$ & $2.04(13)$ \\
\hline 1220 & $12.92(10)$ & $9.32(8)$ & $2.19(12)$ \\
\hline 1233 & $12.86(11)$ & $9.18(8)$ & $2.13(11)$ \\
\hline 1245 & $13.01(12)$ & $9.31(8)$ & $2.22(12)$ \\
\hline
\end{tabular}

a) To whom correspondence should be addressed. Electronic mail: matthew.rowles@curtin.edu.au 
Table SV. Instantaneous and average linear thermal expansion coefficients for $\mathrm{SmCoO}_{3}$ at all temperatures.

\begin{tabular}{|c|c|c|c|c|c|c|}
\hline Temp. (K) & $\begin{array}{l}\text { ILTEC_a } \\
\left(\mathrm{x} 10^{-6} \mathrm{~K}^{-1}\right)\end{array}$ & $\begin{array}{l}\text { ILTEC_b } \\
\left(\mathrm{x} 10^{-6} \mathrm{~K}^{-1}\right)\end{array}$ & $\begin{array}{l}\text { ILTEC_C } \\
\left(\mathrm{x} 10^{-6} \mathrm{~K}^{-1}\right)\end{array}$ & $\begin{array}{l}\text { ALTEC_a } \\
\left(\mathrm{x} 10^{-6} \mathrm{~K}^{-1}\right)\end{array}$ & $\begin{array}{l}\text { ALTEC_b } \\
\left(\mathrm{x} 10^{-6} \mathrm{~K}^{-1}\right)\end{array}$ & $\begin{array}{l}\text { ALTEC_C } \\
\left(\mathrm{x} 10^{-6} \mathrm{~K}^{-1}\right)\end{array}$ \\
\hline 315 & $5.8(6)$ & $9.3(10)$ & $9.9(10)$ & $4.6(5)$ & $9.2(10)$ & $9.6(10)$ \\
\hline 332 & $8.2(5)$ & $11.3(6)$ & $12.0(7)$ & $5.8(3)$ & $10.2(6)$ & $10.7(6)$ \\
\hline 350 & $11.0(4)$ & $13.3(5)$ & $14.1(5)$ & $6.8(3)$ & $10.7(4)$ & $11.4(4)$ \\
\hline 368 & $14.2(4)$ & $15.2(4)$ & 16.1(4) & $7.9(2)$ & $11.4(3)$ & 12.1(3) \\
\hline 386 & $17.4(4)$ & $16.9(4)$ & $17.9(4)$ & $9.2(2)$ & $11.9(3)$ & $12.7(3)$ \\
\hline 403 & $20.7(4)$ & $18.4(3)$ & $19.5(4)$ & $10.5(2)$ & $12.6(2)$ & 13.4(3) \\
\hline 421 & $24.0(4)$ & $19.8(3)$ & $20.9(3)$ & $11.70(19)$ & $13.1(2)$ & $13.9(2)$ \\
\hline 439 & 27.1(4) & 21.1(3) & $22.2(3)$ & 13.22(19) & 13.84(19) & $14.7(2)$ \\
\hline 455 & $30.0(4)$ & 22.3(3) & 23.4(3) & $14.57(18)$ & $14.45(18)$ & 15.31(19) \\
\hline 473 & $32.8(4)$ & $23.5(3)$ & $24.4(3)$ & $16.00(18)$ & $15.07(17)$ & 15.93(18) \\
\hline 490 & $35.4(4)$ & $24.6(3)$ & $25.4(3)$ & $17.33(18)$ & $15.69(16)$ & $16.53(17)$ \\
\hline 507 & $37.9(4)$ & $25.7(2)$ & $26.4(3)$ & 18.67(18) & $16.29(15)$ & $17.13(16)$ \\
\hline 524 & $40.4(4)$ & $26.8(2)$ & $27.5(2)$ & 19.96(17) & $16.88(15)$ & 17.68(15) \\
\hline 541 & $42.9(4)$ & $27.9(2)$ & $28.5(2)$ & 21.12(17) & $17.42(14)$ & $18.20(15)$ \\
\hline 558 & $45.5(3)$ & 29.1(2) & $29.6(2)$ & 22.44(17) & $17.99(14)$ & $18.78(14)$ \\
\hline 575 & $48.0(3)$ & $30.2(2)$ & $30.7(2)$ & 23.73(17) & $18.60(13)$ & $19.37(14)$ \\
\hline 591 & $50.4(3)$ & $31.3(2)$ & $31.6(2)$ & $24.96(17)$ & 19.16(13) & 19.90(13) \\
\hline 608 & $52.4(3)$ & $32.2(2)$ & $32.4(2)$ & $26.22(17)$ & 19.74(13) & $20.49(13)$ \\
\hline 624 & $53.8(3)$ & $33.0(2)$ & $32.9(2)$ & $27.56(17)$ & $20.37(12)$ & $21.08(13)$ \\
\hline 640 & $54.5(3)$ & $33.40(19)$ & 33.12(19) & 28.67(17) & $20.88(12)$ & $21.53(12)$ \\
\hline 656 & $54.4(3)$ & 33.48(19) & 33.02(18) & 29.84(17) & $21.43(12)$ & $22.04(12)$ \\
\hline 673 & $53.3(3)$ & 33.13(18) & $32.52(17)$ & $30.86(16)$ & 21.91(12) & $22.47(12)$ \\
\hline 689 & $51.2(3)$ & $32.30(16)$ & $31.58(16)$ & $31.88(16)$ & $22.44(11)$ & $22.95(12)$ \\
\hline 705 & $48.4(2)$ & 31.11(15) & $30.30(15)$ & $32.47(16)$ & $22.74(11)$ & 23.19(11) \\
\hline 721 & $45.2(2)$ & 29.77(14) & $28.90(14)$ & $33.10(16)$ & $23.09(11)$ & 23.50(11) \\
\hline 737 & 42.09(19) & $28.50(13)$ & 27.58(13) & 33.35(15) & $23.22(11)$ & $23.59(11)$ \\
\hline 752 & $39.42(17)$ & 27.52(12) & $26.55(12)$ & 33.63(15) & $23.40(10)$ & 23.71(10) \\
\hline 768 & $37.34(16)$ & 26.91(11) & 25.88(11) & $33.60(14)$ & $23.39(10)$ & 23.67(10) \\
\hline 783 & $35.67(15)$ & $26.54(11)$ & $25.45(10)$ & $33.87(14)$ & 23.62(10) & $23.86(10)$ \\
\hline 799 & $34.19(14)$ & $26.23(10)$ & $25.10(10)$ & 33.81(13) & $23.62(9)$ & 23.82(9) \\
\hline 814 & $32.70(13)$ & 25.82(10) & $24.68(10)$ & 34.02(13) & 23.87(9) & 24.02(9) \\
\hline 830 & $31.13(12)$ & 25.23(9) & 24.11(9) & 33.88(13) & $23.86(9)$ & 23.97(9) \\
\hline 845 & $29.49(11)$ & $24.49(9)$ & 23.42(9) & $33.96(12)$ & 24.03(9) & 24.10(9) \\
\hline 860 & $27.88(10)$ & 23.71(8) & 22.69(8) & $33.73(12)$ & 23.95(8) & 23.99(9) \\
\hline 875 & $26.37(9)$ & 22.94(8) & 21.96(8) & $33.70(12)$ & 24.05(8) & $24.06(8)$ \\
\hline 889 & 24.96(8) & 22.18(7) & 21.24(7) & 33.53(11) & 24.04(8) & 24.03(8) \\
\hline 904 & 23.74(8) & $21.54(7)$ & $20.63(7)$ & 33.33(11) & 24.01(8) & 23.98(8) \\
\hline
\end{tabular}

a) To whom correspondence should be addressed. Electronic mail:

matthew.rowles@curtin.edu.au 


\begin{tabular}{|l|l|l|l|l|l|l|}
\hline 919 & $22.75(7)$ & $21.06(7)$ & $20.18(6)$ & $33.13(11)$ & $23.96(8)$ & $23.91(8)$ \\
\hline 934 & $21.97(7)$ & $20.74(7)$ & $19.87(6)$ & $32.86(10)$ & $23.87(7)$ & $23.80(7)$ \\
\hline 948 & $21.20(7)$ & $20.38(6)$ & $19.53(6)$ & $32.79(10)$ & $23.96(7)$ & $23.86(7)$ \\
\hline 963 & $20.37(6)$ & $19.91(6)$ & $19.10(6)$ & $32.51(10)$ & $23.86(7)$ & $23.75(7)$ \\
\hline 977 & $19.54(6)$ & $19.39(6)$ & $18.64(5)$ & $32.32(9)$ & $23.85(7)$ & $23.71(7)$ \\
\hline 991 & $18.71(5)$ & $18.85(5)$ & $18.16(5)$ & $32.09(9)$ & $23.79(7)$ & $23.65(7)$ \\
\hline 1005 & $17.88(5)$ & $18.28(5)$ & $17.65(5)$ & $31.90(9)$ & $23.76(7)$ & $23.60(7)$ \\
\hline 1019 & $17.11(5)$ & $17.76(5)$ & $17.18(5)$ & $31.65(9)$ & $23.68(7)$ & $23.52(7)$ \\
\hline 1034 & $16.49(4)$ & $17.37(5)$ & $16.83(5)$ & $31.37(9)$ & $23.57(6)$ & $23.40(6)$ \\
\hline 1048 & $15.99(4)$ & $17.09(5)$ & $16.58(4)$ & $31.14(8)$ & $23.51(6)$ & $23.33(6)$ \\
\hline 1061 & $15.55(4)$ & $16.87(4)$ & $16.39(4)$ & $30.93(8)$ & $23.45(6)$ & $23.26(6)$ \\
\hline 1076 & $15.15(4)$ & $16.71(4)$ & $16.26(4)$ & $30.68(8)$ & $23.36(6)$ & $23.17(6)$ \\
\hline 1089 & $14.82(4)$ & $16.59(4)$ & $16.19(4)$ & $30.47(8)$ & $23.30(6)$ & $23.10(6)$ \\
\hline 1103 & $14.53(4)$ & $16.51(4)$ & $16.15(4)$ & $30.23(7)$ & $23.23(6)$ & $23.02(6)$ \\
\hline 1116 & $14.25(3)$ & $16.41(4)$ & $16.10(4)$ & $30.03(7)$ & $23.19(6)$ & $22.98(6)$ \\
\hline 1130 & $13.96(3)$ & $16.31(4)$ & $16.03(4)$ & $29.78(7)$ & $23.08(6)$ & $22.87(5)$ \\
\hline 1142 & $13.61(3)$ & $16.14(4)$ & $15.88(4)$ & $29.64(7)$ & $23.09(5)$ & $22.88(5)$ \\
\hline 1156 & $13.19(3)$ & $15.88(4)$ & $15.66(4)$ & $29.40(7)$ & $23.00(5)$ & $22.78(5)$ \\
\hline 1169 & $12.75(3)$ & $15.57(4)$ & $15.39(4)$ & $29.22(7)$ & $22.97(5)$ & $22.75(5)$ \\
\hline 1181 & $12.32(3)$ & $15.26(3)$ & $15.13(3)$ & $29.04(7)$ & $22.92(5)$ & $22.71(5)$ \\
\hline 1195 & $12.01(3)$ & $15.06(3)$ & $14.98(3)$ & $28.79(6)$ & $22.82(5)$ & $22.60(5)$ \\
\hline 1207 & $11.83(3)$ & $14.98(3)$ & $14.94(3)$ & $28.60(6)$ & $22.74(5)$ & $22.53(5)$ \\
\hline 1220 & $11.67(3)$ & $14.88(3)$ & $14.86(3)$ & $28.45(6)$ & $22.75(5)$ & $22.55(5)$ \\
\hline 1233 & $11.51(2)$ & $14.73(3)$ & $14.70(3)$ & $28.25(6)$ & $22.68(5)$ & $22.48(5)$ \\
\hline 1245 & $11.44(2)$ & $14.66(3)$ & $14.63(3)$ & $28.04(6)$ & $22.59(5)$ & $22.39(5)$ \\
\hline
\end{tabular}

a) To whom correspondence should be addressed. Electronic mail: matthew.rowles@curtin.edu.au 